\title{
THE JORDAN-HÖLDER THEOREM AND PREFRATTINI SUBGROUPS OF FINITE GROUPS
}

\author{
by A. BALLESTER-BOLINCHES and L. M. EZQUERRO
}

(Received 26 January, 1994)

Introduction. All groups considered are finite. In recent years a number of generalizations of the classic Jordan-Hölder Theorem have been obtained (see [7], Theorem A.9.13): in a finite group $G$ a one-to-one correspondence as in the JordanHölder Theorem can be defined preserving not only $G$-isomorphic chief factors but even their property of being Frattini or non-Frattini chief factors. In [2] and [13] a new direction of generalization is presented: the above correspondence can be defined in such a way that the corresponding non-Frattini chief factors have the same complement (supplement).

In this paper we present a necessary and sufficient condition, named (JH), for a set $\mathbf{X}$ of monolithic maximal subgroups of a group $G$ to verify this wider version of the Jordan-Hölder Theorem: the Jordan-Hölder bijection can be defined in such a way that the corresponding chief factors are $G$-isomorphic, have a supplement in $\mathbf{X}$ at the same time and, in this case, it can be chosen to be a common supplement in $\mathbf{X}$. It is remarkable that $(\mathrm{JH})$ is not only a sufficient condition but is indeed necessary.

On the other hand, W. Gaschütz introduced in [10] a conjugacy class of subgroups of a finite soluble group called prefrattini subgroups. They form a characteristic conjugacy class of subgroups which cover the Frattini chief factors and avoid the complemented ones. These results were generalized by Hawkes [11] and Förster [8] in the soluble case. In [1], the authors introduced the concept of a system of maximal subgroups. These systems can be used to select maximal subgroups in order to define prefrattini subgroups similar to those of Gaschütz, Hawkes and Förster in the general non-soluble case. They enjoy most of the properties of the soluble case except the Cover and Avoidance Property and conjugacy. In fact, conjugacy characterizes solubility.

Another generalization of Gaschütz's work in the soluble universe is due to Kurzweil [12]. He introduced the $H$-prefrattini subgroups of a soluble group $G$, where $H$ is a subgroup of $G$. The $H$-prefrattini subgroups are conjugate in $G$ and they have the Cover and Avoidance Property; if $H=1$ they coincide with the classical prefrattini subgroups of Gaschütz and if $\mathfrak{F}$ is a saturated formation and $H$ is an $\mathfrak{\wp}$-normalizer of $G$ the $H$-prefrattini subgroups are those described by Hawkes.

Tomkinson in [14] extended the results of Gaschütz and Hawkes to a class $\mathfrak{U}$ of locally finite groups with a satisfactory Sylow structure. The intersection of $\mathfrak{U}$ with the class of all finite groups is just the class of all finite soluble groups.

Our aim here is to present all the results of the finite universe in a more unified setting. Our approach is based on Tomkinson's ideas. Using a property, denoted by $\left({ }^{*}\right)$, which is slightly stronger than $(\mathrm{JH})$, we can define $\mathbf{X}$-prefrattini subgroups of a finite

The authors have been supported by Proyecto PB 90-0414-C03-03 of DGICYT, Ministerio de Educación y Ciencia of Spain. The second author has been also supported by Proyecto "Acciones de grupos finitos" of Gobierno de Navarra.

AMS subject classification: Primary 20D30 and 20E28; secondary $20 \mathrm{D} 10$.

Glasgow Math. J. 37 (1995) 265-277. 
group; they enjoy most of the properties of the classical ones and with them we obtain factorizations similar to those of [11] and [1]. This approach also includes the generalizations of Brandis and Kurzweil.

Notation and terminology is standard and can be found in [7]. In particular we note that a group is primitive if it has a core-free maximal subgroup. The class of all primitive groups is composed of the disjoint union of three subclasses, namely the class of primitive groups with abelian socle, the class of primitive groups with a unique non-abelian minimal normal subgroup and the class of primitive groups with exactly two minimal non-abelian normal subgroups. If $M$ is a maximal subgroup of a group $G$ and $G /$ core $_{G}(M)$ is a monolithic primitive group, that is, a group with a unique minimal normal subgroup, then $M$ is called a monolithic maximal subgroup of $G$.

It is assumed that the reader is familiar with the theory of Schunck classes and formations and with the definitions of normalizer and prefrattini subgroup in [1] and its corresponding results.

First definitions and some useful lemmas. Let us begin with a result which partially appears in [4].

LEMMA 1. Let $G$ be a group and suppose that $Z, U, V, W$ are normal subgroups of $G$ such that $Z=U V$ and $U \cap V=W$.

(i) If $Z / U$ is complemented in $G$ by $M$ then $V / W$ is complemented in $G$ by $M$;

(ii) moreover, if $M$ complements $Z / U$ and $S$ complements $U / W$ then $(M \cap S) V$ complements $Z / V$;

(iii) part (i) holds in terms of supplements.

When $V / W$ is a non-abelian chief factor of $G$ we can say even more:

(iv) the set of monolithic supplements of $V / W$ in $G$ coincides with the set of monolithic supplements of $Z / U$ in $G$;

(v) moreover, if $U / W$ is an abelian chief factor of $G$ then the, possibly empty, set of complements of $U / W$ in $G$ coincides with the set of complements of $Z / V$ in $G$.

Proof. Parts (i-iii) are in [4], Hilfssatz 2.4.

For the remainder of the lemma we can suppose without loss of generality that $W=1$ and $V$ is a non-abelian minimal normal subgroup of $G$.

(iv) If $M$ is a monolithic supplement of $V$ in $G$ then $G=M V$ and the group $G / \operatorname{core}_{G}(M)$ is a monolithic primitive group with non-abelian socle. Write $M_{G}=$ core $_{G}(M)$. Then we have $\operatorname{Soc}\left(G / M_{G}\right)=M_{G} V / M_{G}$ and $C_{G}(V)=C_{G}\left(M_{G} V / M_{G}\right)=M_{G}$. Hence $U \leq C_{G}(V)=M_{G} \leq M$. Then $G=M Z$ with $U \leq Z \cap M$ and $M$ is a monolithic supplement of $Z / U$ in $G$. Conversely, if $M$ is a monolithic supplement of $Z / U$ in $G$, then, by (iii), $M$ supplements $V$ in $G$.

(v) Suppose that $V$ is non-abelian and $U$ is an abelian minimal normal subgroup of $G$ complemented by $M$. Then $C_{G}(U)=U$ core $_{G}(M)$ and then $Z=U \times\left(Z \cap\right.$ core $\left._{G}(M)\right)$. This implies that $V=Z^{\prime}=Z \cap \operatorname{core}_{G}(M)$. Then $V \leq M$ and $M$ complements $Z / U$. On the other hand, if $U$ is a Frattini chief factor of $G$, it is clear by (iii) that $Z / V$ must be a Frattini chief factor of $G$. So the (possibly empty) set of complements in $G$ of $U$ coincides with the set of complements of $Z / V$ in $G$. 
Definition. Given a set $\mathbf{X}$ of maximal subgroups of a group $G$, if a chief factor $H / K$ of $G$ has a supplement in $\mathbf{X}$ then it is called an $\mathbf{X}$-supplemented chief factor; otherwise $H / K$ is called an $\mathbf{X}$-Frattini chief factor.

Let $M_{1}$ and $M_{2}$ be two maximal subgroups of the group $G$ such that $C_{1}=$ core $_{G}\left(M_{1}\right) \neq$ core $_{G}\left(M_{2}\right)=C_{2}$ and both complementing an abelian chief factor $H / K$ of $G$. Set $M=\left(M_{1} \cap M_{2}\right) H$. Write $C=C_{G}(H / K)$. Clearly $C=C_{1} H=C_{2} H$. Notice that $C_{1} /\left(C_{1} \cap C_{2}\right) \cong_{G} H / K$. The primitive group associated to $H / K$ and to $C_{1} /\left(C_{1} \cap C_{2}\right)$, i.e. the semidirect product $A=[H / K](G / C)$, is isomorphic to $G / C_{i}$. Now $M$ complements $C_{1} /\left(C_{1} \cap C_{2}\right)$ and then $A \cong G /$ core $_{G}(M)$. Let us calculate core ${ }_{G}(M)$. Clearly $\left(C_{1} \cap\right.$ $\left.C_{2}\right) H \leq \operatorname{core}_{G}(M) \leq C$. Moreover we have $C /\left(C_{1} \cap C_{2}\right) H=C_{1} H /\left(C_{1} \cap C_{2}\right) H \cong_{G}$ $C_{1} /\left(C_{1} \cap C_{2}\right)$ is a chief factor of $G$ and then core ${ }_{G}(M)=H\left(C_{1} \cap C_{2}\right)=D$. Obviously any maximal subgroup $T$ of $G$ such that core ${ }_{G}(T)=D$ complements the chief factors $C / D$ and $C_{i} /\left(C_{1} \cap C_{2}\right), i=1,2$.

Definitions. (i) A set $\mathbf{X}$ of monolithic maximal subgroups of a group $G$ is said to be $J H$-solid if it satisfies the following condition:

$(\mathrm{JH})$ If $M_{1}, M_{2} \in \mathbf{X}$ with $C_{1}=\operatorname{core}_{G}\left(M_{1}\right) \neq$ core $_{G}\left(M_{2}\right)=C_{2}$ and both complement an abelian chief factor $H / K$ of $G$ then there exists $M \in \mathbf{X}$ such that core $_{G}(M)=\left(C_{1} \cap C_{2}\right) H$.

(ii) A set $\mathbf{X}$ of monolithic maximal subgroups of a group $G$ is said to be weakly solid (or $w$-solid) if it satisfies the following condition:

$\left({ }^{*}\right)$ If $M_{1}, M_{2} \in \mathbf{X}$ with $C_{1}=\operatorname{core}_{G}\left(M_{1}\right) \neq$ core $_{G}\left(M_{2}\right)=C_{2}$ and both complement an abelian chief factor $H / K$ of $G$, then $M=\left(M_{1} \cap M_{2}\right) H \in \mathbf{X}$.

(iii) A set $\mathbf{X}$ of maximal subgroups of the group $G$ is said to be solid if it satisfies (*) and whenever a chief factor is $\mathbf{X}$-supplemented, then all its supplements are in $\mathbf{X}$.

(iv) A solid set $\mathbf{X}$ of maximal subgroups of the group $G$ is said to be characteristically solid, or $c$-solid, if $\mathbf{X}^{\alpha}=\mathbf{X}$ for all $\alpha \in \operatorname{Aut}(G)$.

Clearly, if $\mathbf{X}_{1}$ and $\mathbf{X}_{2}$ are JH-solid (resp. w-solid) sets of maximal subgroups of $G$ then $\mathbf{X}_{1} \cap \mathbf{X}_{2}$ is also JH-solid (resp. w-solid).

The following results turn out to be crucial in proving our main results. Their proofs are straightforward.

Lemma 2. If $\mathbf{X}$ is a JH-solid (w-solid, solid) set of maximal subgroups of a group $G$ and $N$ is a normal subgroup of $G$ then the set $\mathbf{X} / N=\{M / N: M \in \mathbf{X}, N \leq M\}$ is a JH-solid ( $w$-solid, solid) set of maximal subgroups of $G / N$.

Lemma 3. Suppose that $M$ is a maximal subgroup of a group $G$ supplementing a minimal normal subgroup $N$ of $G: G=M N$. Let $H / K$ be a supplemented chief factor of $M$ with $N \cap M \leq K$. Then $H N / K N$ is a supplemented chief factor of $G$ and there exists a one-to-one correspondence between the supplements of $H / K$ in $M$ and the supplements of $H N / K N$ in $G$ such that

(i) if $T$ is a supplement of $H / K$ in $M$, then $T N$ is a supplement of $H N / K N$ in $G$;

(ii) core $_{M}(T) N=\operatorname{core}_{G}(T N)$;

(iii) if $S$ is a supplement of $H N / K N$ in $G$, then $S \cap M$ is a supplement of $H / K$ in $M$;

(iv) $\operatorname{core}_{G}(S) \cap M=\operatorname{core}_{M}(S \cap M)$.

Lemma 4. Suppose that $M$ is a maximal subgroup of a group $G$ supplementing $a$ minimal normal subgroup $N$ of $G: G=M N$. Let $\mathbf{X}$ be a JH-solid (resp. w-solid, solid) set 
of maximal subgroups of $G$. Then the set $\mathbf{X} \cap M=\{S \cap M: N \leq S \in \mathbf{X}\}$ is a JH-solid (resp. $w$-solid, solid) set of maximal subgroups of $M$.

Moreover, if $\varphi$ is the isomorphism between $G / N$ and $M /(N \cap M)$ then we have that $(\mathbf{X} / N)^{\varphi}=(\mathbf{X} \cap M) /(M \cap N)$.

Clearly, Lemmas 3 and 4 are meaningful when $G \neq N$, i.e. when $G$ is not a non-abelian simple group.

\section{A Jordan-Hölder-type theorem.}

TheOREM A. Let $G$ be a group and $\mathbf{X}$ a set of monolithic maximal subgroups of $G$.

(a) Let $K$ and $H$ be two normal subgroups of $G$ such that $K<H$. Suppose that

$$
\begin{gathered}
K=N_{0} \leq N_{1} \leq \ldots \leq N_{n}=H \\
K=M_{0} \leq M_{1} \leq \ldots \leq M_{m}=H
\end{gathered}
$$

are parts of two chief series of $G$ between $K$ and $H$.

Then $n=m$ and if $\mathbf{X}$ is $J H$-solid, there exists $\sigma \in \operatorname{Sym}(n)$ such that the following holds:

(i) $N_{i} / N_{i-1} \cong_{G} M_{i^{\sigma}} / M_{i^{\sigma}-1}$;

(ii) $N_{i} / N_{i-1}$ is $\mathbf{X}$-Frattini if and only if $M_{i^{\sigma}} / M_{i^{\sigma}-1}$ is $\mathbf{X}$-Frattini;

(iii) If $N_{i} / N_{i-1}$ is $\mathbf{X}$-supplemented (resp. complemented) in $G$, then there exists a maximal subgroup $C \in \mathbf{X}$ of $G$ such that $C$ supplements (resp. complements) both $N_{i} / N_{i-1}$ and $M_{i} / M_{i^{0}-1}$.

(b) Conversely, suppose that for any pair $K, H$ of normal subgroups of $G$ such that $K<H$ there exists a bijection between the chief factors of any two chief series of $G$ between $K$ and $H$ satisfying the three above conditions ( $i$-ii-iii).

Then $\mathbf{X}$ is a JH-solid set of maximal subgroups of $G$.

Proof. (a) We use induction on $|G|$. By means of a standard argument we may assume that $K=1, N_{1} \neq M_{1}$ and $H=N_{1} \times M_{1}$ for the minimal normal subgroups $N_{1}$ and $M_{1}$.

Following the notation of Lemma 1 we write $U=M_{1}, V=N_{1}$ and $H=Z=U \times V$.

By Lemma 1 (iii-iv) the only situation we have to consider is when $Z$ is abelian. Therefore, suppose that $Z$ is abelian. If $U$ is an $\mathbf{X}$-Frattini chief factor, then so is $Z / V$ by Lemma 1. In this case, if $Z / U$ is also an $\mathbf{X}$-Frattini chief factor, then $V$ must be $\mathbf{X}$-Frattini. Otherwise, if $Z / U$ is $\mathbf{X}$-complemented by $M$, then $V$ is $\mathbf{X}$-complemented by $M$ too, by Lemma 1 . So in both cases the correspondence of $U$ with $Z / V$ and $Z / U$ with $V$ applies.

Thus we can suppose that $U$ and $V$ are $\mathbf{X}$-complemented respectively by, say, $M$ and $S$. Notice that if $Z / U$ and $Z / V$ are $\mathbf{X}$-complemented, then the bijection is clear by Lemma 1 again. So it remains to consider the case where $Z / V$ is an $\mathbf{X}$-Frattini chief factor of $G$. Since $M \in \mathbf{X}$ and $M$ complements $U$, we have $V \ddagger M$ and then $M$ complements $V$. Suppose that, in this case, there exists $T \in \mathbf{X}$ complementing $Z / U$. Then $T$ also complements $V$. Notice that $T, M \in \mathbf{X}$, both complement the abelian subgroup $V$, $U \ddagger$ core $_{G}(M)=M_{G}$ and $U \leq$ core $_{G}(T)=T_{G}$. By the property $(\mathrm{JH})$, there exists $Q \in \mathbf{X}$ such that core $_{G}(Q)=\left(M_{G} \cap T_{G}\right) V$. If $Z \leq Q$, then $Z=Z \cap$ core $_{G}(Q)=V\left(M_{G} \cap T_{G} \cap\right.$ $Z)=V\left(M_{G} \cap U\right)=V$, a contradiction. Hence $Q$ complements $Z / V$, which we have supposed to be $X$-Frattini. From this contradiction we deduce that $Z / U$ must be an 
X-Frattini chief factor of $G$. The correspondence of $U$ with $V$ and $Z / U$ with $Z / V$ applies in this case.

(b) Suppose that we have $M_{1}, M_{2} \in \mathbf{X}$, both complementing the abelian chief factor $H / K$ of $G$ and $C_{1}=\operatorname{core}_{G}\left(M_{1}\right) \neq$ core $_{G}\left(M_{2}\right)=C_{2}$. Write $C=C_{G}(H / K)$. By induction on the order of $G$ we can assume that $C_{1} \cap C_{2}=1$. This implies that $H$ and the $C_{i}$ are minimal normal subgroups of $G$ and indeed $C=C_{i} \times H=C_{1} \times C_{2}, i=1,2$. Hence we have that

$$
1<C_{1}<C \quad 1<H<C
$$

are parts of two chief series of $G$ below $C$. By hypothesis there exists a bijection between the chief factors of both satisfying (i), (ii) and (iii). Notice that the chief factors of the first are both $\mathbf{X}$-complemented and that $C / C_{1}$ and $C / H$ cannot have a common complement in $G$. Hence there exists $M \in \mathbf{X}$ such that $M$ complements $C / H$ and $C_{1}$. Write $D=$ core $_{G}(M)$. Since $C_{1} D=C$, we have $H \leq D<C$ and then $D=H$. Hence $\mathbf{X}$ verifies the property (JH) and $\mathbf{X}$ is JH-solid.

COROLlary. If $\mathbf{X}$ is a JH-solid set of maximal subgroups of a group $G$ and all chief factors $H / K_{i}, i=1, \ldots, n$, of $G$ are $\mathbf{X}$-supplemented, and $\bigcap_{i=1}^{n} K_{i}=K$, then every chief factor between $K$ and $H$ is $\mathbf{X}$-supplemented.

Proof. Denote $K^{j}=\bigcap_{i=1}^{j} K_{i}$ and $K^{0}=H$. Then

$$
K=K^{n} \leq K^{n-1} \leq \ldots \leq K^{0}=H
$$

is a piece of a chief series of $G$. If $K^{i} / K^{i+1}$ is a chief factor of $G$ (i.e. if $K^{i} \neq K^{i+1}$ ), then $H=K^{i} K_{i+1}$ and $K^{i} / K^{i+1} \cong_{G} H / K_{i+1}$. Apply Lemma 1 and if $M$ is an $\mathbf{X}$-supplement of $H / K_{i+1}$ in $G$, then $M$ is an $\mathbf{X}$-supplement of $K^{i} / K^{i+1}$ in $G$. We deduce that all chief factors in the above series are $\mathbf{X}$-supplemented. Now apply Theorem $\mathrm{A}$ to conclude the proof.

As a consequence, the definition of solid set of maximal subgroups made by Tomkinson in [14] coincides with ours for a finite soluble group $G$.

Remark. Given a modular lattice $\mathscr{L}$, J. Lafuente in [13] introduced the concept of $M$-set in $\mathscr{L}$ and he proved a general Jordan-Hölder theorem in modular lattices with an $M$-set.

In our context, if $\mathbf{X}$ is a JH-solid set of maximal subgroups of a group $G$ then the set $\mathcal{M}$ of all $\mathbf{X}$-supplemented chief factors of $G$ is an $M$-set in the modular lattice $\mathcal{N}$ of all normal subgroups of $G$. With this, our Theorem $\mathrm{A}(\mathrm{a})$ is indeed a particular case of Lafuente's version of the Jordan-Hölder theorem. Nevertheless, the arguments we have presented here to prove Theorem A(a) are exactly those to prove that $\mathcal{M}$ is an $M$-set. It must be observed that in [13] it is proved that the bijection mentioned in Theorem $A(a)$ is unique.

Conversely, suppose that $\mathbf{X}$ is a set of monolithic maximal subgroups of $G$ such that the set of $\mathbf{X}$-supplemented chief factors $\mathcal{M}$ is an $M$-set in $\mathcal{N}$. If $M_{1}, M_{2} \in \mathbf{X}$ both complement the same abelian chief factor $H / K$ and $C_{1}=\operatorname{core}_{G}\left(M_{1}\right) \neq$ core $_{G}\left(M_{2}\right)=C_{2}$ then $C / C_{i}, C_{i} /\left(C_{1} \cap C_{2}\right), i=1,2$, and $H\left(C_{1} \cap C_{2}\right) /\left(C_{1} \cap C_{2}\right)$ are $\mathbf{X}$-supplemented chief 
factors, i.e. they belong to $\mathcal{M}$. The condition of $M$-set implies that $C / H\left(C_{1} \cap C_{2}\right) \in \mathcal{M}$ and hence there exists $M \in \mathbf{X}$ such that $M$ supplements $C / H\left(C_{1} \cap C_{2}\right)$. Notice that core $_{G}(M)=H\left(C_{1} \cap C_{2}\right)$ and hence $\mathbf{X}$ is a $J H$-solid set of maximal subgroups of $G$.

On page 361 of [13], Lafuente wonders about the precise condition on any set of maximal subgroups $\mathbf{X}$ of $G$ such that the set of chief factors of $G$ complemented by at least one element $U \in \mathbf{X}$ is an $M$-set in $\mathcal{N}$. What we have shown above is that $J H$-solid sets of maximal subgroups are the answer to Lafuente's question for sets of monolithic maximal subgroups if complementation is changed to supplementation.

\section{EXAMPLES.}

(1) Consider the group $G=\left\langle a, b, c: a^{5}=b^{5}=c^{4}=[a, b]=1, a^{c}=a^{2}, b^{c}=b^{3}\right\rangle$. Denote $A=\langle a\rangle$ and $B=\langle b\rangle$. The sets $\mathbf{X}_{1}=\left\{A P: P \in \operatorname{Syl}_{2}(G)\right\}$ and $\mathbf{X}_{2}=\left\{B P: P \in \operatorname{Syl}_{2}(G)\right\}$ are both solid. If $\alpha$ is the automorphism of $G$ such that $a^{\alpha}=b, b^{\alpha}=a, c^{\alpha}=c^{3}$ then $\mathbf{X}_{1}^{\alpha}=\mathbf{X}_{2}$.

(2) Consider a subgroup $L$ of a group $G$; the set $\mathbf{X}_{L}$ of all monolithic maximal subgroups of $G$ containing $L$ is $w$-solid.

(3) The set $\operatorname{Max}(G)$, of all maximal subgroups of a group $G$, is $c$-solid.

Let $M$ be a monolithic maximal subgroup of $G$. Recall that the normal index of $M$ in $G$ is defined by Deskins in [6] to be $\eta(G, M)=\left|\operatorname{Soc}\left(G / \operatorname{core}_{G}(M)\right)\right|$.

(4) The following families of maximal subgroups of a group $G$ are $w$-solid:

(i) for a fixed prime $p$, the set $\mathbf{X}_{p}$ of all monolithic maximal subgroups $M$ of $G$ such that $|G: M|$ is a $p$-power; (In fact, if $G$ is soluble, then $\mathbf{X}_{p}$ is solid. However this is not true in the non-soluble case; in $G=\operatorname{Alt}(5)$ the set $\mathbf{X}_{5}$ is composed of all maximal subgroups isomorphic to Alt(4) and clearly it is not solid.)

(ii) for a fixed set of primes $\pi$, the set $\mathbf{X}_{\pi}$ of all monolithic maximal subgroups $M$ of $G$ such that $|G: M|$ is a $\pi$-number;

(iii) the set of all monolithic maximal subgroups of $G$ of composite index in $G$;

(iv) the set of all monolithic maximal subgroups $M$ of the group $G$ such that $\eta(G, M) \neq|G: M|$;

(5) Let $\mathfrak{H}$ be a class of groups. A maximal subgroup $M$ of a group $G$ is said to be Se-normal in $G$ if $G /$ core $_{G}(M) \in \mathfrak{S}$; otherwise it is said to be $\mathfrak{S}_{e}$-abnormal. The set $\operatorname{Max}(G)_{\mathfrak{W}}^{n}$, of all monolithic $\mathscr{F e}_{\mathrm{c}}$-normal maximal subgroups of $G$, and its complementary set $\operatorname{Max}(G)_{\mathfrak{5}}^{a}$, of all monolithic $\mathscr{S}_{e}$-abnormal maximal subgroups of $G$, are both $w$-solid.

In general the set of all $\mathfrak{S}$-normal maximal subgroups of $G$ and the set of all

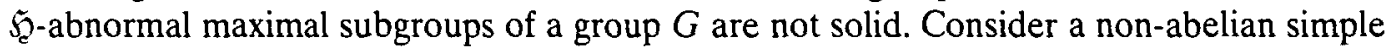
group $S$ and let $M$ be a maximal subgroup of $S$. Form the direct product $G=S_{1} \times S_{2}$, where $S_{1} \cong S \cong S_{2}$ and take the Schunck class $\mathcal{S}_{2}$ of all $G$-perfect groups. The minimal normal subgroup $S_{1}$ is supplemented by $M_{1} \times S_{2}$ which is $\mathfrak{S}_{e}$-normal and by the diagonal subgroup which is $\mathfrak{S}_{\mathfrak{c}}$-abnormal.

In fact, given any group $G$, if $\mathfrak{S}$ is a Schunck class, then the sets $\operatorname{Max}(G)_{\mathscr{W}}^{n}$ and $\operatorname{Max}(G)_{\mathfrak{W}}^{a}$ are solid if and only if the boundary $b(\mathfrak{S})$ is composed of monolithic primitive groups

(6) Given a $w$-solid set $\mathbf{X}$ of maximal subgroups of a group $G$, then the sets $\mathbf{X}_{\mathfrak{Q}}^{a}=\mathbf{X} \cap\left(\operatorname{Max}(G)_{\mathfrak{W}}^{a}\right)$ and $\mathbf{X}_{\mathfrak{G}}^{n}=\mathbf{X} \cap\left(\operatorname{Max}(G)_{\mathfrak{b}}^{n}\right)$ are $w$-solid.

(7) Every system of maximal subgroups of a group $G$ (cf. [1]) is a $w$-solid set. In particular, if $G$ is a soluble group and $\Sigma$ a Hall system of $G$, the set $\mathscr{S}(\Sigma)$ composed of all 
maximal subgroups $M$ of $G$ such that $\Sigma$ reduces into $M$ is a system of maximal subgroups of $G$ and then is a $w$-solid set.

\section{Prefrattini subgroups.}

Definition. Let $G$ be a group, $\mathscr{S}$ a system of maximal subgroups of $G$ and $\mathbf{X}$ a $\boldsymbol{w}$-solid set of maximal subgroups of $G$. Suppose that $\mathbf{X} \cap \mathscr{S} \neq \varnothing$ and form the subgroup

$$
W(G, \mathbf{X}, \mathscr{P})=\bigcap_{M \in \mathbf{X} \cap \mathscr{S}} M .
$$

We say that $W(G, \mathbf{X}, \mathscr{Y})$ is the $\mathbf{X}$-prefrattini subgroup of $G$ associated with $\mathscr{S}$. If $\mathbf{X} \cap \mathscr{S}=\varnothing$ we put $W(G, \mathbf{X}, \mathscr{S})=G$.

Denote by $\operatorname{Pref}(\mathbf{X}, G)$ the set of all $\mathbf{X}$-prefrattini subgroups of $G$ which are proper subgroups of $G$, i.e.

$$
\operatorname{Pref}(\mathbf{X}, G)=\{W(G, \mathbf{X}, \mathscr{P}): \mathbf{X} \cap \mathscr{S} \neq \varnothing\} .
$$

Obviously if $\mathbf{X}$ is solid, $\operatorname{Pref}(\mathbf{X}, G)$ is the set of all $\mathbf{X}$-prefrattini subgroups of $G$.

EXAMPLES.

(1) Let $G$ be the group of Example 1. Denote by $N=A B$ the Sylow 5-subgroup of $G$. If $P_{i}, i=1,2,3$, are three different Sylow 2-subgroups of $G$, the set $\mathbf{X}=\left\{A P_{1}, B P_{2}\right\}$ is $w$-solid. Consider the Hall-systems $\Sigma_{i}=\left\{N, P_{i}\right\}, i=1,2,3$. Then $W\left(G, \mathbf{X}, \mathscr{S}\left(\Sigma_{1}\right)\right)=A P_{1}$ and $W\left(G, \mathbf{X}, \mathscr{S}\left(\Sigma_{2}\right)\right)=B P_{2}$. On the other hand, $\mathbf{X} \cap \mathscr{P}\left(\Sigma_{3}\right)=\varnothing$ and $W\left(G, \mathbf{X}, \mathscr{Y}\left(\Sigma_{3}\right)\right)=G$.

(2) If $G$ is a soluble group, then the set $\operatorname{Pref}\left(G, \mathbf{X}_{L}\right)$ is the set of all $L$-prefrattini subgroups introduced by Kurzweil in [12].

(3) If $G$ is a soluble group, then the $\operatorname{Max}(G)$-prefrattini subgroups are the usual prefrattini subgroups introduced by Gaschütz in [10].

(4) In the universe of all $p$-soluble groups the $\mathbf{X}_{p}$-prefrattini subgroups are the $p$-prefrattini subgroups studied by Brandis in [4]. [1].

(5) The $\operatorname{Max}(G)_{5}^{a}$-prefrattini subgroups are the $\mathfrak{S}$-prefrattini subgroups defined in

Next we see that these prefrattini subgroups are preserved under epimorphic images.

THeOREM B. Consider a group $G, \mathbf{X}$ a w-solid set of maximal subgroups of $G, \mathscr{S}$ a system of maximal subgroups of $G$ and $W=W(G, \mathbf{X}, \mathscr{Y})$.

(i) Let $1=G_{0}<G_{1}<\ldots<G_{n}=G$ be a chief series of $G$; suppose that $\mathbf{X} \cap \mathscr{S} \neq \varnothing$ and write $I=\left\{i: 1 \leq i \leq n\right.$ such that $G_{i} / G_{i-1}$ is $\mathbf{X} \cap \mathscr{Y}_{\text {-supplemented }\} \text {; then }}$

$$
W=\bigcap_{i \in I}\left\{S_{i}: S_{i} \text { is an } \mathbf{X} \text {-supplement of } G_{i} / G_{i-1} \text { in } \mathscr{S}\right\} \text {. }
$$

(ii) If $N$ is a normal subgroup of $G$ then $\{W N / N: W$ is an $\mathbf{X}$-prefrattini subgroup of $G$ \} is the set of all $\mathbf{X} / N$-prefrattini subgroups of $G / N$ and in fact $W(G / N, \mathbf{X} / N$, $\mathscr{S N} / N)=W N / N$.

Proof. (i) Denote $D=\bigcap_{i \in J}\left\{S_{i}: S_{i}\right.$ is an $\mathbf{X}$-supplement of $G_{i} / G_{i-1}$ in $\left.\mathscr{S}\right\}$. It is clear that $W(G, \mathbf{X}, \mathscr{P}) \leq D$. On the other hand notice that if $M$ and $S$ are both $\mathbf{X}$-supplements in $\mathscr{S}$ of the same chief factor $H / K$, then core ${ }_{G} M \neq$ core $_{G} S$. This forces $H / K$ to be abelian and then $T=(M \cap S) H \in \mathbf{X} \cap \mathscr{S}$. Since $K=S \cap H=M \cap H$, we have $S \cap T=(S \cap M)(S \cap$ 
$H)=(S \cap M) K=S \cap M=M \cap T$. Therefore the other possible maximal subgroups in $\mathbf{X} \cap \mathscr{S}$ do not change the intersection and equality holds.

(ii) Let $N$ be a minimal normal subgroup of $G$. If $\mathbf{X} / N \cap \mathscr{S} / N=\varnothing$, then $W(G / N$, $\mathbf{X} / N, \mathscr{S} N / N)=G / N$. If $\mathbf{X} \cap \mathscr{S}=\varnothing$, then $W(G, \mathbf{X}, \mathscr{S})=G$ and there is nothing to prove. If $\mathbf{X} \cap \mathscr{S} \neq \varnothing$, then, by condition $\left({ }^{*}\right)$ and the definition of systems of maximal subgroups, we have $\mathbf{X} \cap \mathscr{S}=\{M\}$ with $G=M N$. Since $W(G, \mathbf{X}, \mathscr{Y})=M$, the result follows.

Suppose now that $\mathbf{X} / N \cap \mathscr{S N} / N \neq \varnothing$. Take a chief series of $G$ through $N$ : $1=G_{0}<G_{1}=N<\ldots<G_{n}=G$. If there exists an $\mathbf{X}$-supplement $S_{1}$ of $N$ in $\mathscr{Y}$, we have $W=W(G, \mathbf{X}, \mathscr{S})=S_{1} \cap\left(\bigcap_{i>1} S_{i}\right)$, where $N \leq S_{i}$ for all $i>1$. Clearly $W N=\bigcap_{i>1} S_{i}$ and we have the result. If no $\mathbf{X}$-supplement in $N$ is in $\mathscr{Y}$, then $N \leq W(G, \mathbf{X}, \mathscr{S})$ and again we have the result.

If $N$ is not a minimal normal subgroup of $G$, consider a chief factor $N / K$ of $G$ and apply the above argument to the group $G / K$.

Remark. Theorem B does not hold when $\mathbf{X}$ is only a $J H$-solid set. This is the reason why we introduce our prefrattini subgroups associated to $w$-solid sets and not to $J H$-solid sets of maximal subgroups. The following example shows this.

Denote by $V$ the elementary abelian group of order 9 . The cyclic group $C$ of order 2 acts on $V$ by inversion. Form the semidirect product $G=[V] C$ and write $V=\langle a, b\rangle$ and $C=\langle z\rangle$. Consider the set of maximal subgroups $\mathbf{X}=\left\{M_{1}=\langle a, z\rangle, M_{2}=\langle b, a z\rangle, M_{3}=\right.$ $\left.\langle a b, z\rangle, M_{4}=\left\langle a^{2} b, z\right\rangle\right\}$ which is $J H$-solid but not $w$-solid. Denote by $A$ the normal subgroup $\langle a\rangle$. The group $G$ is soluble and in fact every system of maximal subgroups of $G$ is associated with a unique Hall-system of $G$ (see [1], Cor.2.11). Consider the following Hall-systems: $\Sigma_{1}=\{V,\langle z\rangle\}, \Sigma_{2}=\{V,\langle a b z\rangle\}, \Sigma_{3}=\left\{V,\left\langle a^{2} b^{2} z\right\rangle\right\}$. Subindexing in the obvious way the prefrattini subgroups, we have $W_{1}=M_{1} \cap M_{2}=\langle z\rangle, W_{2}=M_{1}, W_{3}=M_{3} \cap M_{4}=$ $\left\langle a^{2} b^{2} z\right\rangle$.

Consider the chief series $1<A<V<G$ of $G$. Notice that if we consider the system of maximal subgroups associated with $\Sigma_{3}$, Theorem $\mathrm{B}(\mathrm{i})$ does not hold.

Consider now the quotient group $G / A \cong \operatorname{Sym}(3)$. Then $\mathbf{X} / A=\left\{M_{1} / A\right\}$ and the $\mathbf{X} / A$-prefrattini subgroups of $G$ are $M_{1} / A$, associated to $\Sigma_{1} A / A$, and $G / A$ otherwise. Clearly $W_{2} A / A$ and $W_{3} A / A$ are not $\mathbf{X} / A$-prefrattini subgroups of $G / A$ and Theorem $\mathrm{B}$ (ii) does not hold either.

PROPOSITION. Let $G$ be a group and $\mathbf{X}$ a c-solid set of maximal subgroups. Then the set of $\mathbf{X}$-prefrattini subgroups is a characteristic class of subgroups of $G$.

In Example 1 we see that this is not true in general. The set of $\mathbf{X}_{1}$-prefrattini subgroups is exactly $\mathbf{X}_{1}$ which is not closed under automorphisms (Compare with [14]).

Definition. The intersection of all $\mathbf{X}$-prefrattini subgroups is called the $\mathbf{X}$-Frattini subgroup of $G$. It will be denoted by $\Phi_{\mathbf{X}}(G)$.

Proposition. If $\mathbf{X}$ is a solid set of maximal subgroups of a group $G$ and $W$ is an $\mathbf{X}$-prefrattini subgroup of a group $G$ then $\operatorname{core}_{G}(W)=\Phi_{\mathbf{X}}(G)$.

Proof. Let $\mathscr{S}$ be a system of maximal subgroups of $G$. Consider $W=W(G, \mathbf{X}, \mathscr{S})$.

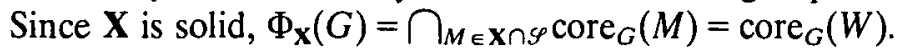

The classical Frattini subgroup of a group $G, \Phi(G)$, is clearly the $\operatorname{Max}(G)$-Frattini subgroup of $G$. The set $\operatorname{Max}(G)_{\mathfrak{M}}^{a}$ of all self-normalizing maximal subgroups of $G$ is solid 
and the $\operatorname{Max}(G)_{\mathfrak{R}}^{a}$-Frattini subgroup is denoted by $L(G)$ in [3]. The set $\operatorname{Max}(G)_{\Re}^{n}$ of all normal maximal subgroups of $G$ is also solid and Bechtell denotes the $\operatorname{Max}(G)_{\mathfrak{M}}^{n}$-Frattini subgroup by $R(G)$. Following this notation, if $\mathfrak{S}$ is a Schunck class we can denote by $L_{\mathfrak{G}}(G)$ the $\operatorname{Max}(G)_{\mathfrak{5}}^{a}$-Frattini subgroup of $G$ and similarly $R_{\mathfrak{Q}}(G)$ will be the $\operatorname{Max}(G)_{\mathfrak{S}^{-}}^{n}$ Frattini subgroup of $G$.

In Theorem (4.4) of [1] it is proved that if $G$ is a group and $₹$ is a saturated formation, then $L_{\Re}(G)=Z_{\Re}(G \bmod \Phi(G))$. Now a description of $R_{\Re}(G)$ can be obtained.

Proposition. Let $G$ be a group. If $\mathfrak{F}$ is a saturated formation and $\mathscr{S}$ is a system of

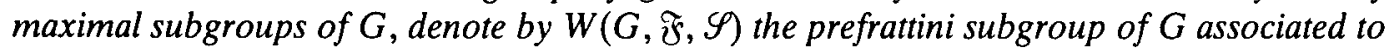
$\mathscr{S}$ and defined by the solid set of all $§-n o r m a l$ maximal subgroups of $G$. Then

$$
\text { core }_{G} W(G, \mathfrak{F}, \mathscr{S})=R_{\Re}(G)=\Phi\left(G \bmod G^{\Im}\right) \text {. }
$$

Proof. First notice that $G^{\Re}$ is contained in every $\mathfrak{\Im}$-normal maximal subgroup of $G$ and if $G \in \mathfrak{F}$, then every maximal subgroup of $G$ is $\mathfrak{₹}$-normal. Therefore, $R_{\mathfrak{F}}(G) / G^{\mathfrak{F}}=$ $R_{\Re}\left(G / G^{\Re}\right)=\Phi\left(G / G^{\Re}\right)$. Since $G^{\mathfrak{S}} \leq$ core $_{G} W(G, \mathfrak{f}, \mathscr{S})$, by Theorem $\mathrm{B}$, we have $W(G, \mathfrak{F}, \mathscr{S}) / G^{\Re}=W\left(G / G^{\Re}, \mathfrak{\Im}, \mathscr{S} / G^{\Re}\right)=W\left(G / G^{\mathfrak{\Im}}, \mathscr{Y} / G^{\Im}\right)$ and, taking cores, we have $\operatorname{core}_{G}(W(G, \mathfrak{F}, \mathscr{S})) / G^{\mathfrak{F}}=\Phi\left(G / G^{\Im}\right)$.

In [11] an elegant theorem of factorization of prefrattini subgroups of soluble groups is proved. There, Hawkes makes a strong use of the Cover and Avoidance property. Later, in [1], we obtained a similar factorization in the general non-soluble universe but in this situation every appearance of the Cover and Avoidance property had to be removed, showing that Hawkes' factorization does not really require this CAP-property. Following these ideas we obtain here a similar result.

THEOREM C. Let $G$ be a group and $\mathfrak{F}$ a Schunck class of the form $\mathfrak{S}_{\mathfrak{Z}}=\mathrm{E}_{\Phi} \mathfrak{\mho}$ where $\mathfrak{\mho}$ is a formation. Consider a system of maximal subgroups $\mathscr{S}$ of $G$. Then, if $\mathbf{X}$ is a w-solid set of maximal subgroups of $G$, we have

$$
W\left(G, \mathbf{X}_{\mathscr{\wp}}^{a}, \mathscr{P}\right)=D W(G, \mathbf{X}, \mathscr{T})
$$

where $D$ is an $\mathfrak{\mathfrak { C }}$-normalizer of $G$ associated with $\mathscr{S}$.

Proof. Denote $W\left(\mathbf{X}_{\mathfrak{L}}^{a}\right)=W\left(G, \mathbf{X}_{\mathfrak{G}}^{a}, \mathscr{S}\right)$ and $W(\mathbf{X})=W(G, \mathbf{X}, \mathscr{S})$. By Theorem 4.9 of [1], we know that $D$ is contained in every $\mathfrak{S}_{\mathfrak{E}}$-abnormal maximal subgroup of $G$ in $\mathscr{S}$. Hence $\langle D, W(\mathbf{X})\rangle \leq W\left(\mathbf{X}_{\mathfrak{G}}^{a}\right)$. If $G \in \mathfrak{F}$, then $G=D$ and $\mathbf{X}_{\mathfrak{S}}^{a}=\varnothing$. Thus, $W\left(\mathbf{X}_{\mathfrak{W}}^{a}\right)=G=D$.

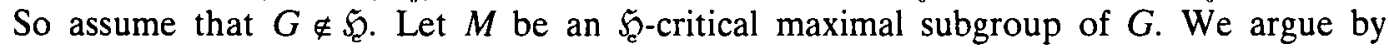
induction on the order of $G$. Obviously we can suppose that $\Phi(G)=1$. This means that $M$ supplements a minimal normal subgroup $N$ of $G$. If $G$ is a simple group, then every maximal subgroup of $G$ is $\mathfrak{S}$-abnormal and then $W\left(G, \mathbf{X}_{\mathscr{f}}^{a}, \mathscr{S}\right)=W(G, \mathbf{X}, \mathscr{P})$. So the Theorem is true in this case. Hence we can assume that $N$ is a proper subgroup of $G$ and $N \cap M \neq M$. Now $\mathbf{X} / N$ and $\mathbf{X}_{\tilde{q}}^{a} / N$ are $w$-solid sets of maximal subgroups of $G / N$ and $\mathbf{X}_{\mathfrak{W}}^{a} / N=(\mathbf{X} / N)_{\tilde{w}}^{a}$. By Theorem B we have $W(\mathbf{X}) N / N=W(G / N, \mathbf{X} / N, \mathscr{S} / N)$ and $W\left(\mathbf{X}_{\mathfrak{5}}^{a}\right) N / N=W\left(G / N,(\mathbf{X} / N)_{\mathfrak{5}}^{a}, \mathscr{S} / N\right)$. By induction, we have $W(\mathbf{X}) D N=W\left(\mathbf{X}_{\mathfrak{5}}^{a}\right) N$.

If $N$ is an $\mathbf{X}$-Frattini minimal normal subgroup or $N$ is $\mathbf{X}$-supplemented but no $\mathbf{X}$-supplement of $N$ in $G$ is in $\mathscr{S}$, then $N \leq W(\mathbf{X}) \leq W\left(\mathbf{X}_{\mathfrak{\wp}}^{a}\right)$ and the result follows. So we can suppose that $M \in \mathbf{X}$ (and then $M \in \mathbf{X}_{\mathscr{\wp}}^{a}$ ) and $M \in \mathscr{Y}$.

Denote by $\varphi$ the isomorphism $G / N \cong M /(N \cap M)$. We have $(\mathscr{Y} / N)^{\varphi}$ is a system of 
maximal subgroups of $M /(N \cap M)$ and there exists a system of maximal subgroups $\mathscr{S}_{M}$ of $M$ such that $\mathscr{S}_{M} /(N \cap M)=(\mathscr{Y} / N)^{\varphi}$ and $\mathscr{S} \cap M \subseteq \mathscr{S}_{M}$. Now $\left((\mathbf{X} / N)_{\mathfrak{G}}^{a}\right)^{\varphi}=\left(\mathbf{X}_{\mathfrak{S}}^{a} / N\right)^{\varphi}=$ $\left(\mathbf{X}_{\mathfrak{S}}^{a} \cap M\right) /(M \cap N)=[(\mathbf{X} \cap M) /(M \cap N)]_{\mathfrak{w}}^{a}$ by Lemmas 3 and 4 . Then $W\left(M, \mathbf{X}_{\mathfrak{5}}^{a} \cap M\right.$, $\left.\mathscr{S}_{M}\right)(N \cap M)=W\left(\mathbf{X}_{\mathfrak{G}}^{a}\right) N \cap M=W\left(\mathbf{X}_{\mathfrak{q}}^{a}\right)(N \cap M)$. Therefore $W\left(M, \mathbf{X} \cap M, \quad \mathscr{S}_{M}\right)(N \cap$ $M)=W(\mathbf{X}) N \cap M=W(\mathbf{X})(N \cap M)$. But by Theorem B(i), we know that $W(\mathbf{X})$ is the intersection of supplements in $\mathscr{S}$. of the $\mathbf{X}$-supplemented chief factors of a chief series of $G$. If we take this chief series through the normal subgroup $N$ and consider $M$ in this intersection, we have $N \cap M \leq W(\mathbf{X}) \leq W\left(\mathbf{X}_{\mathfrak{\wp}}^{a}\right)$. Then $W\left(M, \mathbf{X}_{\tilde{q}}^{a} \cap M, \mathscr{S}_{M}\right)(N \cap M)=$ $W\left(\mathbf{X}_{\mathfrak{G}}^{a}\right)$. Hence $W\left(M, \mathbf{X} \cap M, \mathscr{S}_{M}\right)(N \cap M)=W(\mathbf{X})$. It is clear that $D$ is the $\mathfrak{S}_{2}$-normalizer of $M$ associated to $\mathscr{S}_{M}$. By induction, $W\left(M, \mathbf{X}_{\mathscr{\Phi}}^{a} \cap M, \mathscr{S}_{M}\right)=D W\left(M, \mathbf{X} \cap M, \mathscr{S}_{M}\right)$. By taking the product with $N \cap M$ in both sides of the equality we obtain the required factorization.

Inspired by Theorem 5.3 of [14] we have the following factorization involving Se-normal maximal subgroups.

THEOREM D. Let $G$ be a group and $\mathfrak{S}$ a saturated formation. Consider a system of maximal subgroups $\mathscr{S}$ of $G$. Then if $\mathbf{X}$ is a w-solid set of maximal subgroups of $G$ we have

$$
W\left(G, \mathbf{X}_{\mathfrak{b}}^{n}, \mathscr{S}\right)=W(G, \mathbf{X}, \mathscr{S}) G^{\mathfrak{G}} .
$$

Proof. It is clear that $G^{\mathscr{E}} \leq M$ for all $M \in \mathbf{X}_{\mathfrak{b}}^{n}$. Therefore, for every system $\mathscr{S}$ of maximal subgroups of $G$, we have $G^{\mathfrak{G}} \leq W\left(G, \mathbf{X}_{\mathfrak{b}}^{n}, \mathscr{S}\right)$. Since $G / G^{\mathfrak{E}} \in \mathfrak{F}$, we have $\mathbf{X}_{\mathfrak{G}}^{n} / G^{\mathfrak{Q}}=\mathbf{X} / G^{\mathfrak{G}}$. Then

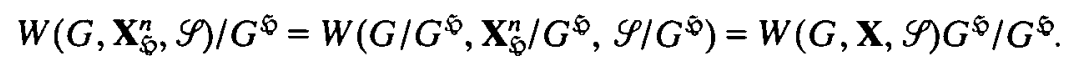

COROllary. Let $G$ be a group and $\mathfrak{S}$ a saturated formation. Consider a system of maximal subgroups $\mathscr{S}$ of $G$. Then, if $\mathbf{X}$ is a w-solid set of maximal subgroups of $G$, we have

$$
G=W\left(G, \mathbf{X}_{\mathfrak{\wp}}^{n}, \mathscr{Y}\right) W\left(G, \mathbf{X}_{\mathfrak{W}}^{a}, \mathscr{Y}\right) .
$$

Proof. Just notice that if $D$ is an $\mathfrak{S}$-normalizer of $G$, then $G=D G^{\mathfrak{W}}$. Now apply the factorizations of Theorems $C$ and $D$.

All classical examples of prefrattini subgroups in the soluble universe, including Kurzweil's, enjoy the conjugacy and the Cover and Avoidance properties. In fact conjugacy of prefrattini subgroups characterizes solubility (see [1] Th. 4.3). The consideration of primitive non-soluble groups, whose core-free maximal subgroups are neither conjugate nor CAP-subgroups causes these properties to fail in the general non-soluble universe.

Our Theorem E shows that, roughly speaking, it can be said that Conjugacy and Cover and Avoidance property of soluble chief factors are equivalent properties for prefrattini subgroups.

Let $G$ be a group and $\mathbf{X}$ a $w$-solid set of maximal subgroups of $G$. Notice that every $\mathbf{X}$-prefrattini subgroup of a group $G$ covers the $\mathbf{X}$-Frattini chief factors of $G$. We will be more precise in the following proposition whose proof we omit.

Proposition. Let $H / K$ be an $\mathbf{X}$-supplemented chief factor of $G$ and $\mathscr{S}$ a system of maximal subgroups of $G$.

(i) If $H / K$ possesses an $\mathbf{X}$-complement in $\mathscr{S}$ then $W(G, \mathbf{X}, \mathscr{S})$ avoids $H / K$. 
(ii) If $H / K$ is abelian and does not possess an $\mathbf{X}$-complement in $\mathscr{S}$ then $W(G, \mathbf{X}, \mathscr{S})$ covers $H / K$.

Corollary. If $\mathbf{X}$ is solid, and $H / K$ is an abelian chief factor of $G$ then $H / K$ is either covered or avoided by every $\mathbf{X}$-prefrattini subgroup of $G$. Moreover, $H / K$ is covered if and only if it is an X-Frattini chief factor of $G$.

Definition. Let $G$ be a group and $\mathbf{X}$ a $w$-solid set of maximal subgroups of $G$. Write $\mathscr{W}=\operatorname{Pref}(\mathbf{X}, G)$. We say that $\mathscr{W}$ satisfies $A C A P$ if whenever $F$ is an abelian chief factor of $G$, then $F$ is either covered or avoided by all $W \in \mathscr{W}$ and $F$ is covered if and only if $F$ is an X-Frattini chief factor of $G$.

Clearly if $\mathscr{W}$ satisfies ACAP, any $W \in \mathscr{W}$ covers all abelian $\mathbf{X}$-Frattini chief factors of $G$ and avoids all abelian $\mathbf{X}$-complemented chief factors.

EXAMPLES.

(a) Given a group $G$, if $\mathbf{X}$ is solid, then $\operatorname{Pref}(\mathbf{X}, G)$ satisfies ACAP.

(b) By ([12], Lemma 1.5) if $G$ is soluble and $L \leq G$ the set $\operatorname{Pref}\left(\mathbf{X}_{L}, G\right)$ of all $L$-prefrattini subgroups of $G$ satisfies ACAP; notice that $\mathbf{X}_{L}$ is $w$-solid.

(c) Let $G$ be the group presented in the remark after Theorem B. The set $\mathbf{X}$ considered there is $\mathrm{JH}$-solid and the $\mathbf{X}$-prefrattini subgroups do not satisfy ACAP. With the same notation, consider $\mathbf{X}_{0}=\left\{\langle a, z\rangle,\langle b, z\rangle,\langle a b, z\rangle,\left\langle a^{2} b, z\right\rangle\right\}$. Then $\mathbf{X}_{0}$ is $w$-solid. Now $W\left(G, \mathbf{X}_{0}, \Sigma_{1}\right)=\langle z\rangle$ and $W\left(G, \mathbf{X}_{0}, \Sigma_{2}\right)=\langle a b, z\rangle$. It is clear that the $\mathbf{X}_{0}$-prefrattini subgroups of $G$ do not satisfy ACAP.

Proposition. Let $G$ be a group, $\mathbf{X}$ a w-solid set of maximal subgroups of $G$ and let $\mathscr{W}=\operatorname{Pref}(\mathbf{X}, G)$. Assume that $\mathscr{W}$ satisfies ACAP. Let $\mathscr{S}_{1}, \mathscr{S}_{2}$ be two systems of maximal subgroups of $G$ and $H / K$ an abelian chief factor of $G$. Then there exists an $\mathbf{X}$-complement of $H / K$ in $\mathscr{S}_{1}$ if and only if there exists an $\mathbf{X}$-complement of $H / K$ in $\mathscr{S}_{2}$.

Proof. Write $\{i, j\}=\{1,2\}$. Suppose that $M_{i}$ is an $\mathbf{X}$-complement of $H / K$ in $\mathscr{S}_{i}$ but for all maximal subgroups $S \in \mathbf{X} \cap \mathscr{S}_{j}$ such that $K \leq S$ we have $H \leq S$. Denote by $W_{k}$ the X-prefrattini subgroup of $G$ associated to $\mathscr{S}_{k}, k=1,2$. It is clear that $W_{i} \leq M_{i}$. Then $K=W_{i} K \cap H$. Since $\mathscr{W}$ satisfies ACAP, we have $K=W_{j} K \cap H$. However $W_{j} K / K$ is the $\mathbf{X} / K$-prefrattini subgroup of $G / K$ associated with $\mathscr{S}_{j} / K$ and then $W_{j} K / K=\bigcap\{S / K: S \in$ $\left.\mathbf{X} \cap \mathscr{S}_{j}, K \leq S\right\}$. Our assumption implies $H / K \leq W_{j} K / K$, a contradiction. So the proposition holds.

THEOREM E. Let $G$ be a group and $\mathbf{X}$ a w-solid set of maximal subgroups of $G$. Let $\mathscr{W}=\operatorname{Pref}\left(\mathbf{X}_{\mathfrak{S}}^{n}, G\right)$, where $\mathbb{S}$ is the class of all soluble groups. Then the following are equivalent:

(i) $W$ satisfies ACAP;

(ii) all subgroups in $\mathscr{W}$ are conjugate in $G$.

Proof. (i) $\Rightarrow$ (ii). Assume that (ii) does not hold and let $G$ be a minimal counterexample. If $H$ is any non-trivial normal subgroup of $G$, then $\mathbf{X} / H$ is a $w$-solid set of maximal subgroups of $G / H$. Notice that $(\mathbf{X} / H)_{\subseteq}^{n}=\mathbf{X}_{\subseteq}^{n} / H$. Moreover $\operatorname{Pref}\left(\mathbf{X}_{\Subset}^{n} / H, G / H\right)$ satisfies ACAP. So, by induction, $\operatorname{Pref}\left(\mathbf{X}_{\subseteq}^{n} / H, G / H\right)$ is a set of conjugate subgroups of $G / H$.

Let $N$ be a minimal normal subgroup of $G$. If $N$ is $\mathbf{X}_{\mathscr{S}}^{n}$-Frattini, then $N$ is covered by 
every $\mathbf{X}_{\mathscr{E}}^{n}$-prefrattini subgroup of $G$. Since the theorem holds in $G / N$, we obtain a contradiction. Then $N$ is $\mathbf{X}_{\mathscr{C}}^{n}$-supplemented. In particular $N$ is $\widetilde{S}$-central and therefore $N$ is abelian. There exists $M \in \mathbf{X}_{\mathscr{E}}^{n}$ such that $G=M N$ and $M \cap N=1$. Let $\mathscr{S}$ be a system of maximal subgroups of $G$ such that $M \in \mathscr{Y}$. Denote by $A$ the $\mathbf{X}_{\mathscr{E}}^{n}$-prefrattini subgroup of $G$ associated to $\mathscr{S}$. It is clear that $A \leq M$. Since by hypothesis $\mathscr{W}$ is not a set of conjugate subgroups of $G$, there exists a system $\mathscr{S}_{0}$ of maximal subgroups of $G$ such that if $A_{0}$ is the $\mathbf{X}_{\subseteq}^{n}$-prefrattini subgroup of $G$ associated to $\mathscr{S}_{0}$, then $A$ and $A_{0}$ are non-conjugate in $G$.

Let $\varphi$ be the isomorphism between $G / N$ and $M$. We have $(\mathbf{X} / N)_{\subseteq}^{n}=\mathbf{X}_{\mathscr{S}}^{n} / N$ and $\left(\mathbf{X}_{\mathscr{S}}^{n} / N\right)^{\varphi}=(\mathbf{X} \cap M)_{\subseteq}^{n}=\mathbf{X}_{\mathscr{S}}^{n} \cap M$. Denote $C=\operatorname{core}_{G}(M)=C_{M}(N)$. Suppose that $C \neq 1$. Since the theorem holds in $G / C$, there exists $x \in G$ such that $A_{0}^{x} C=A C \leq M$. So we can assume that $x=1$. In particular $A_{0} \leq M$. Then $A N \cap M=A$ and $A_{0} N \cap M=A_{0}$ are $(\mathbf{X} \cap M)_{\mathbb{E}}^{n}$-prefrattini subgroups of $M$ and by induction they are conjugate in $M$, a contradiction.

Therefore $C=1$. Since $M$ is 5 -normal, we have $G$ is a primitive soluble group. The solubility of $G$ implies that there exists $g \in G$ such that $\mathscr{S}_{g}=\mathscr{S}$. We apply here the above proposition to conclude that $\mathbf{X} \cap \mathscr{S}=\mathbf{X} \cap \mathscr{S}_{0}=\left(\mathbf{X} \cap \mathscr{S}_{0}\right)^{g}$ and $A=\bigcap\{M: M \in \mathbf{X} \cap \mathscr{S}\}=$ $\bigcap\left\{S: S \in\left(\mathbf{X} \cap \mathscr{S}_{0}\right)^{g}\right\}=\bigcap\left\{S^{8}: S \in \mathbf{X} \cap \mathscr{S}_{0}\right\}=A_{0}^{g}$, final contradiction.

(ii) $\Rightarrow$ (i). Notice that all non-abelian chief factors of $G$ are $\mathbf{X}_{\mathscr{E}}^{n}$-Frattini. This means that $\mathbf{X}_{\mathscr{E}}^{n}$-prefrattini subgroups are conjugate CAP-subgroups. The conclusion follows.

Therefore the Theorem holds.

Corollary. Let $G$ be a soluble group, $\mathbf{X}$ a w-solid set of maximal subgroups of $G$ and $\mathscr{W}=\operatorname{Pref}(\mathbf{X}, G)$. The following statements are equivalent:

(i) $\mathscr{W}$ is a set of conjugate subgroups of $G$, and

(ii) every $W \in \mathscr{W}$ is a CAP-subgroup of $G$ which covers all $\mathbf{X}$-Frattini chief factors of $G$ and avoids the $\mathbf{X}$-complemented ones.

\section{REFERENCES}

1. A. Ballester-Bolinches and L. M. Ezquerro, On maximal subgroups of finite groups, Comm. Algebra 19(8) (1991), 2373-2394.

2. D. W. Barnes, On complemented chief factors of finite soluble groups, Bull. Austral. Math. Soc. 7 (1972), 101-104.

3. H. Bechtell, Pseudo-Frattini subgroups, Pacific J. Math. 14 (1964), 1129-1136.

4. A. Brandis, Moduln und verschränkte Homomorphismen endlicher Gruppen, J. reine angew. Math. 385 (1988), 102-116.

5. R. W. Carter, B. Fischer and T. O. Hawkes, Extreme classes of finite soluble groups, $J$. Algebra 9 (1968), 285-313.

6. W. E. Deskins, On maximal subgroups, Proc. Symp. in Pure Math., Amer. Math. Soc. 1 (1959), 100-104.

7. K. Doerk and T. O. Hawkes, Finite Soluble Groups, (de Gruyter, 1992).

8. P. Förster, Prefrattini subgroups, J. Austral. Math. Soc. (Series A) 34 (1983), 234-247.

9. P. Förster, Chief factors, crowns and the generalised Jordan-Hölder Theorem, Comm. Algebra 16(8) (1988), 1627-1638.

10. W. Gaschütz, Praefrattinigruppen, Arch. Math. 13 (1962), 418-426.

11. T. O. Hawkes, Analogues of Prefrattini subgroups, Proc. Internat. Conf. Theory of Groups, Austral. Nat. Univ. Canberra. (1965), 145-150.

12. H. Kurzweil, Die Praefrattinigruppe in Intervall eines Untergruppenverbandes, Arch. Math. 53 (1989), 235-244. 
13. J. Lafuente, Maximal subgroups and the Jordan-Hölder Theorem, J. Austral. Math. Soc. (Series A) 46 (1989), 356-364.

14. M. J. Tomkinson, Prefrattini subgroups and cover-avoidance properties in $\mathfrak{U}$-groups, Can. J. Math. 27 (1975), 837-851.

Departament d'Àlgebra, UNIVERSITAT DE VALÈNCIA, C/Dr. MOLINER 50, 46100 Burjassot (ValènCia), Spain.

Departamento de Matemática e Informática, Universidad Pública de Navarra, Campus de Arrosadía, 31006 Pamplona, Spain. 